\title{
The Cryogenic AntiCoincidence Detector Project for ATHENA+: An Overview Up to the Present Status
}

\author{
C. Macculi · L. Piro - L. Colasanti - S. Lotti • \\ L. Natalucci - D. Bagliani · M. Biasotti · F. Gatti • \\ G. Torrioli · M. Barbera - T. Mineo · E. Perinati
}

Received: 12 July 2013 / Accepted: 24 February 2014 / Published online: 17 March 2014

(C) Springer Science+Business Media New York 2014

\begin{abstract}
ATHENA+ is a space mission proposal for the next ESA L2-L3 slot. One of the focal plane instruments is the X-ray integral field unit (X-IFU) working in the energy range $0.3-10 \mathrm{keV}$. It is a multi-array based on TES detectors aimed at characterizing faint or diffuse sources (e.g. WHIM or galaxy outskirt). The X-IFU will be able to achieve the required sensitivity if a low background is guaranteed. The studies performed by GEANT4 simulations depict a scenario where the use of an active anticoincidence (AC) is mandatory to reduce the background expected in L2 orbit down to the goal level of $0.005 \mathrm{cts} \mathrm{cm}^{-2} \mathrm{~s}^{-1} \mathrm{keV}^{-1}$. This is possible using a cryogenic anticoincidence (CryoAC) detector placed within a proper optimized environment surrounding the X-IFU. We propose a $2 \times 2$ array of microcalorimeter detectors made by silicon absorber (each of about $1 \mathrm{~cm}^{2}$ and $300 \mu \mathrm{m}$ thick) and sensed by an Ir TES. In order to better understand the involved physics and optimize the performance, we
\end{abstract}

\footnotetext{
C. Macculi $(\varangle) \cdot$ L. Piro $\cdot$ L. Colasanti $\cdot$ S. Lotti $\cdot$ L. Natalucci

INAF/IAPS Roma, Rome, Italy

e-mail: claudio.macculi@iaps.inaf.it

D. Bagliani · M. Biasotti · F. Gatti

Physics Department, Genova University, Genoa, Italy

G. Torrioli

CNR/IFN Roma, Rome, Italy

M. Barbera

Physics and Chemistry Department, Palermo University, Palermo, Italy

T. Mineo

INAF/IASF Palermo, Palermo, Italy

E. Perinati

IAAT Tübingen-D, Tübingen, Germany
} 
have produced several samples featured by different absorber areas, TES size, and QPs $\mathrm{Al}$ collectors. Here we will discuss, as a review, the obtained results and the related impact to the final detector design.

Keywords TES $\cdot$ Silicon · Iridium $\cdot$ Anticoincidence detector $\cdot$ Space

\section{Introduction}

ATHENA+ is an X-ray observatory proposed as a large mission for the ESA science program [1]. It has been conceived to answer some of the most pressing questions in astrophysics for the late 2020s, that can be uniquely addressed with X-ray observations. ATHENA+ will transform our understanding of two major components of the cosmos: the hot universe and the energetic universe. Basically, it will help us in understanding the following fundamental issues: (1) how does ordinary matter assemble into the large scale structures that we see today? and (2) how do black holes grow and shape the universe? To reach the scientific goals, it is necessary to put a high performance X-Ray TES-based spectrometer (X-IFU [2]) at the focal plane of a high performance optics, and this must be coupled to a cryogenic anticoincidence (CryoAC) detector. The aim of this paper is to show what is the development level of the CryoAC project reached so far, and it is structured as follows: in Sect. 2, we will discuss a scientific case which emphasizes the presence of the CryoAC; in Sect. 3 we will show the results so far obtained from the sensors development, what we have to do, and some consideration about the electronics; then the conclusions are reported in Sect. 4.

\section{The Necessity of the CryoAC for ATHENA+ at L2-orbit: An Example}

Considerations of observing efficiency and thermal stability favour the insertion of the ATHENA+ satellite in the L2 orbit. Given the scientific purpose of the mission, the particle background around X-IFU has to be low: the goal is $0.005 \mathrm{cts} \mathrm{cm}^{-2} \mathrm{~s}^{-1} \mathrm{keV}^{-1}$ in the $0.3-10 \mathrm{keV}$ working bandwidth. Much work has been done [3] to model the expected particle background on L2, due to lack of data since no X-ray mission has ever flown there. Starting from the ATHENA case [4] results, we can evaluate what we could expect for the ATHENA+ case by means of the GEANT4 toolkit (see $[3,5]$ and Refs. therein). The structures surrounding X-IFU are quite complex. Towards the instruments, beyond the cryostat Al-walls, there is copper, gold but also niobium which constitutes the magnetic shield to properly operate the TES detectors. So, a primary proton hitting the payload will cross different masses/materials generating secondary particles, mainly electrons, and fluorescence lines. Figure 1, left panel (i.e. the "reduced background") shows how the bkg (background) is reduced by a proper design of the CryoAC coupling to the X-IFU. In particular we show that a further reduction of a factor six in the residual bkg with respect to our previous design (i.e. ATHENA-XMS), can be achieved by: (a) decreasing the distance between the X-IFU and the CryoAC (from $\sim 2 \mathrm{~mm}$ to $\sim 1 \mathrm{~mm}$ ), (b) the application of a thin $(250 \mu \mathrm{m}$ ) kapton layer interposed between the niobium and X-IFU, and (c) moving one of the radiation filters, $100 \mathrm{~nm}$ thick kapton-based, close to the X-IFU detector surface [4]. 


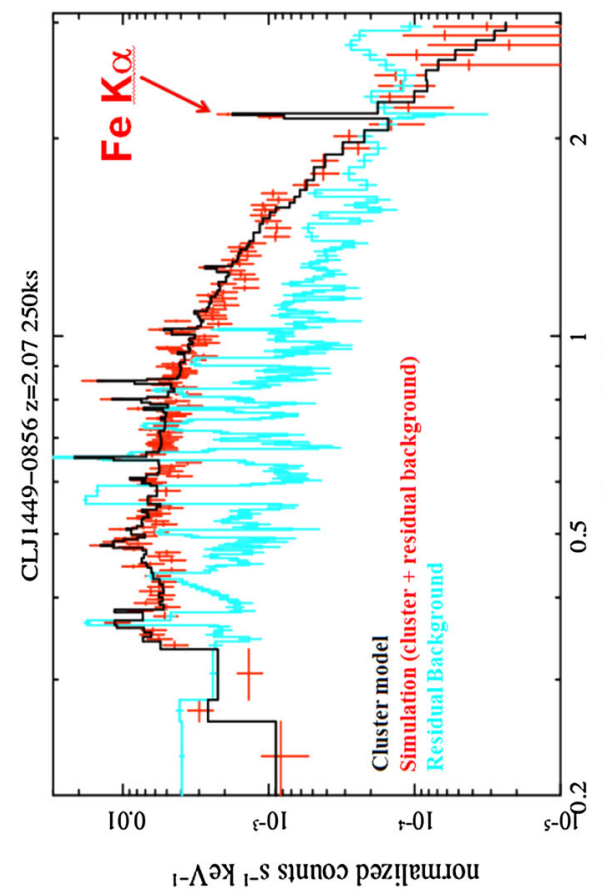

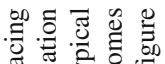

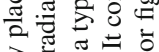

วิำ

낭 bon

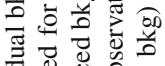

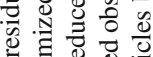

Q

¿

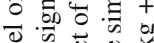

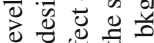

ช

政政

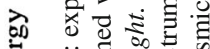

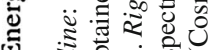

స 0 की

选选 늠

후닙

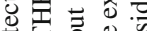

它它导

\&

क्ये

D 2

뭠

䒕运芯芯

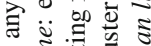

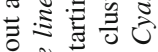

言芯范范

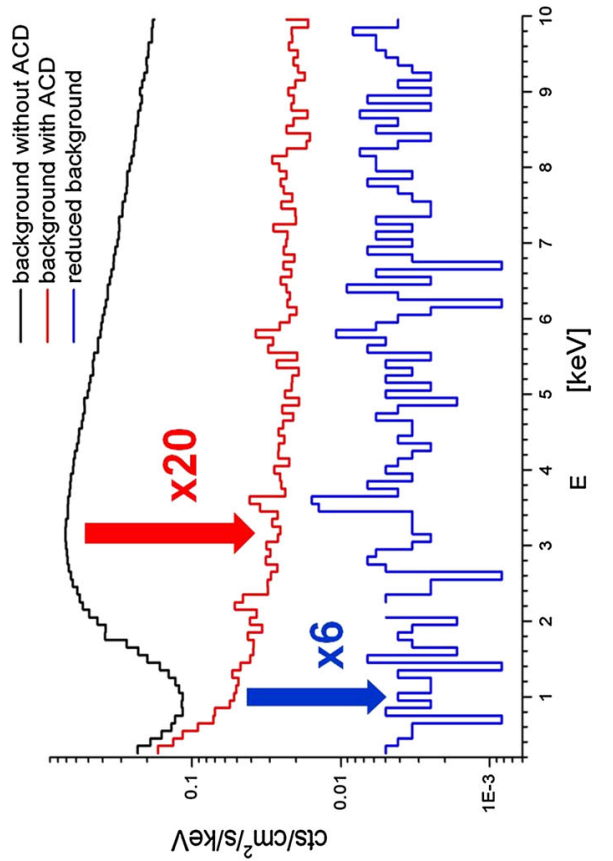

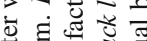

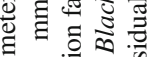

웡

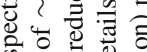

के

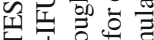

$\dot{x} \div$

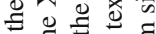

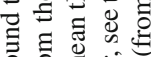

ฮั

ป

폻ㅊ

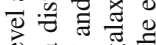

0.

늠 $\times \stackrel{0}{0}$

式完士

焉石

过吾至

过导它

芩

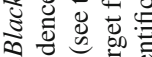

巡苛 志.

$\checkmark$.

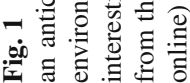


Few words about the kapton layer [4-6]. Assuming as optimized the efficiency of active rejection of the primary particles and of hard secondaries, the residual particle bkg is composed mostly of secondary soft electrons emitted from niobium, which are completely absorbed by X-IFU without activating the CryoAC. The kapton has a high electronic stopping power and at the same time a much lower emission yield of a metal when excited by primary hard protons. Then a thin sheet of kapton interposed between the niobium and the X-IFU fulfills the aim of breaking down the shower of electrons in $0.3-10 \mathrm{keV}$ band which are streaming towards the latter.

To understand what is the effect of this reduced bkg we have simulated an observation of the Galaxy cluster [7] CLJ1449-0856 placed at redshift $\mathrm{z}=2$ (see Fig. 1). The simulated flux is $\mathrm{F}=10^{-15} \mathrm{erg} \mathrm{cm}^{-2} \mathrm{~s}^{-1}$, corresponding to a source luminosity of $\mathrm{L}=7 \cdot 10^{43} \mathrm{erg} \mathrm{s}^{-1}$. For the source we assumed an abundance $=0.3$, and temperature $\mathrm{kT}=2.0 \mathrm{keV}$. We used a source extraction area $=0.2 \operatorname{arcmin}^{2}$, area $=1 \mathrm{~m}^{2}$, focal length $=12 \mathrm{~m}$. For the simulation we used the above source model with our estimated bkg spectrum, consisting of the cosmic diffuse component plus the residual internal particles one. The result is that the cluster model is recovered. Thanks to this clean spectrum coupled with the imaging capability of ATHENA+ it is possible to obtain the cluster redshift, the abundances of several elements, and study the dynamics (shocks, etc.) $[8]$.

\section{The CryoAC Detector: The TES Microcalorimeter and the Electronics}

The CryoAC detector has to be considered as an independent instrument constituted by a $2 \times 2$ pxl TES-array and the related cryogenic (i.e. SQuID) and warm electronics. At present it is foreseen to correlate on ground the CryoAC output used as anticoincidence flag with the X-IFU pulses. As overview, this section will discuss the samples, what we have done, what we have to do, and then conclude with some details of the electronics. The present requirements of the CryoAC are shown in Table 1. We remark that the threshold is $20 \mathrm{keV}$.

\subsection{What We have Done}

Along the programme about the development for CryoAC related to X-ray missions, from IXO [9] to ATHENA+, we have produced and tested several samples of microcalorimeters, based on silicon absorber and sensed by Ir TES [13, 14] (Fig. 2).

We started from a simple sensor (AC-S1) where the Ir TES is deposited onto the Si aborber. From the first results [13] (see Fig. 3 top-left) we understood that the detec-

Table 1 Requirements of the CryoAC for ATHENA+

\begin{tabular}{ll}
\hline Size & $18 \times 18 \mathrm{~mm}^{2}$ (in 4 pixel, each $80 \mathrm{~mm}^{2}$ ) \\
Thickness & $300-500 \mu \mathrm{m}$ \\
Rise time constant & $<30 \mu \mathrm{s}$ \\
Decay time constant & $<300 \mu \mathrm{s}$ (goal) \\
Bandpass & $20 \mathrm{keV}-0.5 \mathrm{MeV}$ \\
\hline
\end{tabular}




\begin{tabular}{|c|c|}
\multicolumn{1}{c}{ AC-S1 } \\
\hline $\begin{array}{c}\text { Area } \\
{\left[\mathbf{m m}^{2}{ }^{2}\right]}\end{array}$ & $\begin{array}{c}\text { Collecting } \\
\text { area }(\%)\end{array}$ \\
\hline 16.5 & 11 \\
\hline
\end{tabular}

AC-S2

\begin{tabular}{|c|c|}
\hline $\begin{array}{c}\text { Area } \\
{\left[\mathbf{m m}^{2}\right]}\end{array}$ & $\begin{array}{c}\text { Collecting } \\
\text { area }(\%)\end{array}$ \\
\hline 100 & 9 \\
\hline
\end{tabular}

AC-S3,4

\begin{tabular}{|c|c|}
\hline $\begin{array}{c}\text { Area } \\
{\left[\mathrm{mm}^{2}\right]}\end{array}$ & $\begin{array}{c}\text { Collecting } \\
\text { area }(\%)\end{array}$ \\
\hline 100 & $\sim 20$ \\
\hline
\end{tabular}

AC-S5

\begin{tabular}{|c|c|}
\hline $\begin{array}{c}\text { Area } \\
\text { [mm }^{2} \text { ] }\end{array}$ & $\begin{array}{c}\text { Collecting } \\
\text { area (\%) }\end{array}$ \\
\hline 42 & $\sim 24$ \\
\hline
\end{tabular}

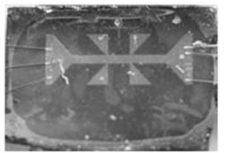

\begin{tabular}{|c|c|}
\hline $\begin{array}{c}\text { Ir TES Area } \\
{\left[\mathrm{mm}^{2}\right]}\end{array}$ & $\begin{array}{c}\text { Abs } \\
\text { Thickness }\end{array}$ \\
\hline 3.7 & $300 \mathrm{um}$ \\
\hline
\end{tabular}

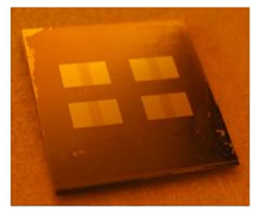

\begin{tabular}{|c|c|}
\hline $\begin{array}{c}\text { Ir TES + Al Area } \\
{\left[\mathrm{mm}^{2}\right]}\end{array}$ & $\begin{array}{c}\text { Abs } \\
\text { Thickness }\end{array}$ \\
\hline $1.5 \times 4-1.5 \times 8$ & $380 \mathrm{um}$ \\
\hline
\end{tabular}

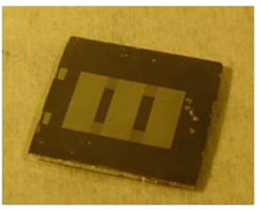

\begin{tabular}{|c|c|}
\hline $\begin{array}{c}\text { Ir TES + Al Area } \\
{\left[\mathbf{m m}^{2}\right]}\end{array}$ & $\begin{array}{c}\text { Abs } \\
\text { Thickness }\end{array}$ \\
\hline $1.5 \times 4-10 \times 3$ & $380 \mathrm{um}$ \\
\hline
\end{tabular}

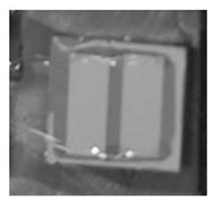

Fig. 2 History of the samples both produced and tested up to now. The tables show some geometric characteristics. The collecting area is the ratio between the sum of $\mathrm{Ir}$ and $\mathrm{Al}$ area to the full absorber area (Color figure online)

tor response was a combination of thermal and athermal pulses [10], so realizing the necessity to increase the collecting efficiency of the athermal phonons which, indeed, we use as a fast anticoincidence flag. To increase such a collecting area usually Al pad (or fins) are adopted $[11,12]$. So we developed the AC-S2, AC-S3, four samples to investigate this issue. Further, due to the good results from our first AC-S1, since we are not concerned about the detector energy resolution, another tentative solution has been pursued to increase only the Ir area (Ir has a specific heat much bigger than the $\mathrm{Al}$ ), rather than to add the Al-fins. So, we developed also the AC-S5 sample, our last tested one. It has been, and yet it is, necessary to develop samples featured by different Al-pad and absorber sizes not only because from data analysis [13,14] we have had to understand several phenomena involving quasiparticles and thermal/athermal phonons, but also because it is still a matter of discussion the development of a 4 or 1-pixel detector as CryoAC. This is the reason why we have produced so different samples, so to gather as much information as possible. At last, we have also developed a model to predict, at the moment, the thermal component of the pulse. Extended discussion about the results so far obtained are presented in Refs. [13,14].

In brief, to be compared with the requirements, here is a summary of the results obtained from this activity by taking into account all the sensors so far tested: (a) size $>20 \mathrm{~mm}^{2}$ (up to $100 \mathrm{~mm}^{2}$ ); (b) "athermal" fast component ( $\sim \mu \mathrm{s}$ ) successfully obtained: rise time consistent with the electronics $\mathrm{L} / \mathrm{R}$ with fast rise $<30 \mu \mathrm{s}$; (c) decay times from $\sim 15 \mu \mathrm{s}$ to $>600 \mu \mathrm{s}$ (pure athermals or combination of $\mathrm{A}+\mathrm{T}$ ); (d) 


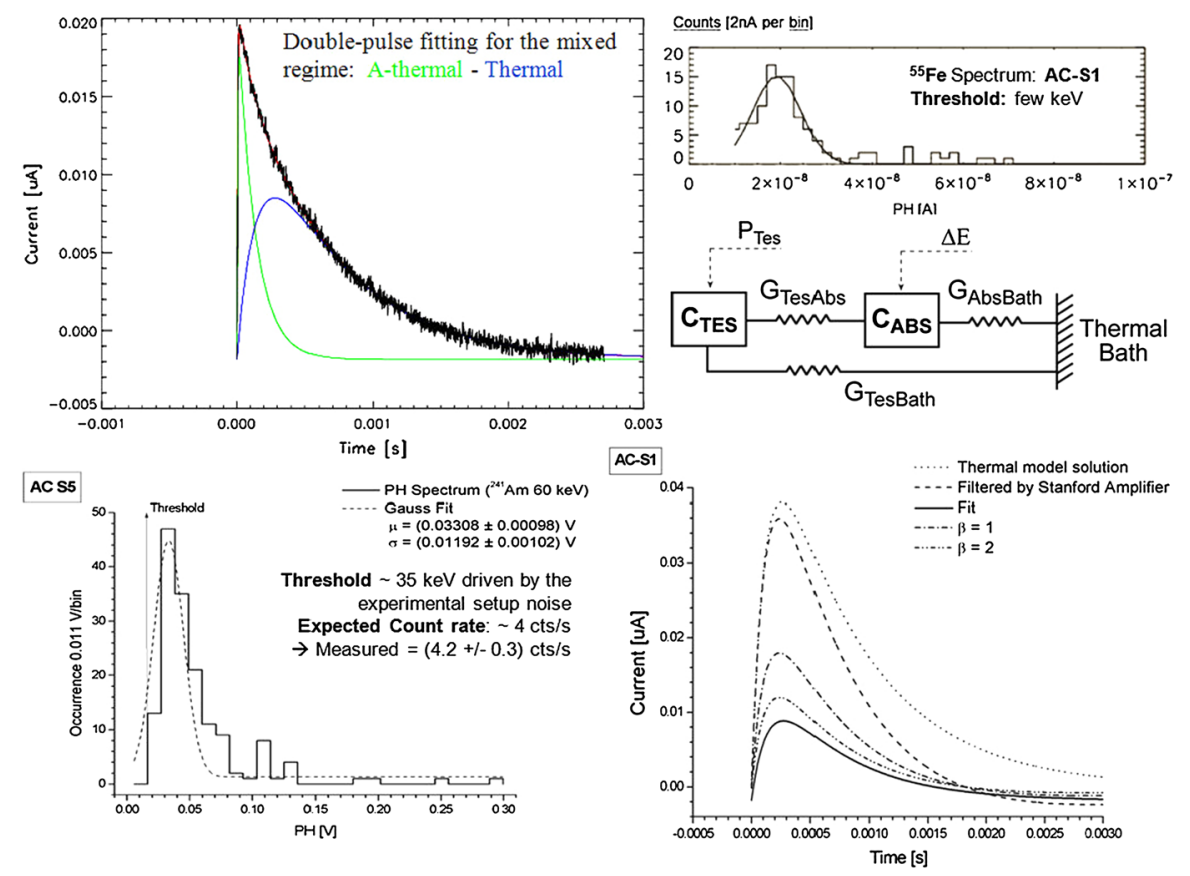

Fig. 3 Results from AC-S1 and AC-S5 samples. From top-left clockwise: (AC-S1) athermal + thermal components from the measured pulse; (AC-S1) spectrum from the ${ }^{55} \mathrm{Fe}$ source $(\sim 6 \mathrm{keV})$; (AC-S1) thermal model used to evaluate the thermal component; (AC-S1) comparison between the expected thermal component from the model dotted, then filtered by the Stanford amplifier dashed, then by taking into account two plausible values for the current sensitivity (not measured), and the thermal component extracted by fitting the pulse on the top-left of this Fig. 3 blue line; spectrum from AC-S5 used to evaluate the observed count rate (illuminated by the ${ }^{241} \mathrm{Am}$ source $-60 \mathrm{keV}$ ) (Color figure online)

thermal component consistent with modelling; (e) low energy threshold: few keV for $16 \mathrm{~mm}^{2}$ (AC-S1), $35 \mathrm{keV}$ for $\sim 50 \mathrm{~mm}^{2}$ (AC-S5 noise-EMI dominated); (f) quantum efficiency: observed count rate compatible with the expected one (AC-S5). Thanks to these results, at the moment our baseline model used for the next samples design, is AC-S1. Figure 3 shows the most important results about the AC-S1, and the obtained quantum efficiency by AC-S5 thanks to the increased athermals collecting area (only increased Ir pads). Few words about the Al-pads present in AC-S2, AC-S3,4 samples (AC-S3,4 has pad size, so collecting area greater than AC-S2). We remind that we add them not only to well-shape the spectrum (i.e. the peak-spread was related to the not-good collection of all the athermal phonons produced [13,14]) but also to increase the quantum efficiency (i.e. observed count rate compatible with the expected one). In the second attempt (i.e. AC-S3), we have detected the recombination of quasiparticles [13], so producing two-pulses families: one is the direct heat into the TES, the other one is related to heat released after the recombination. We have also observed that the greater the detected energy, the faster the pulse (see Fig. 4). As presented in Ref. [13], we have understood the energy response behaviour. Poor energy response was essentially produced by a collection of phonons through the Al fingers, which degrades quickly above mm sizes. This is also the reason why at the moment AC-S1 


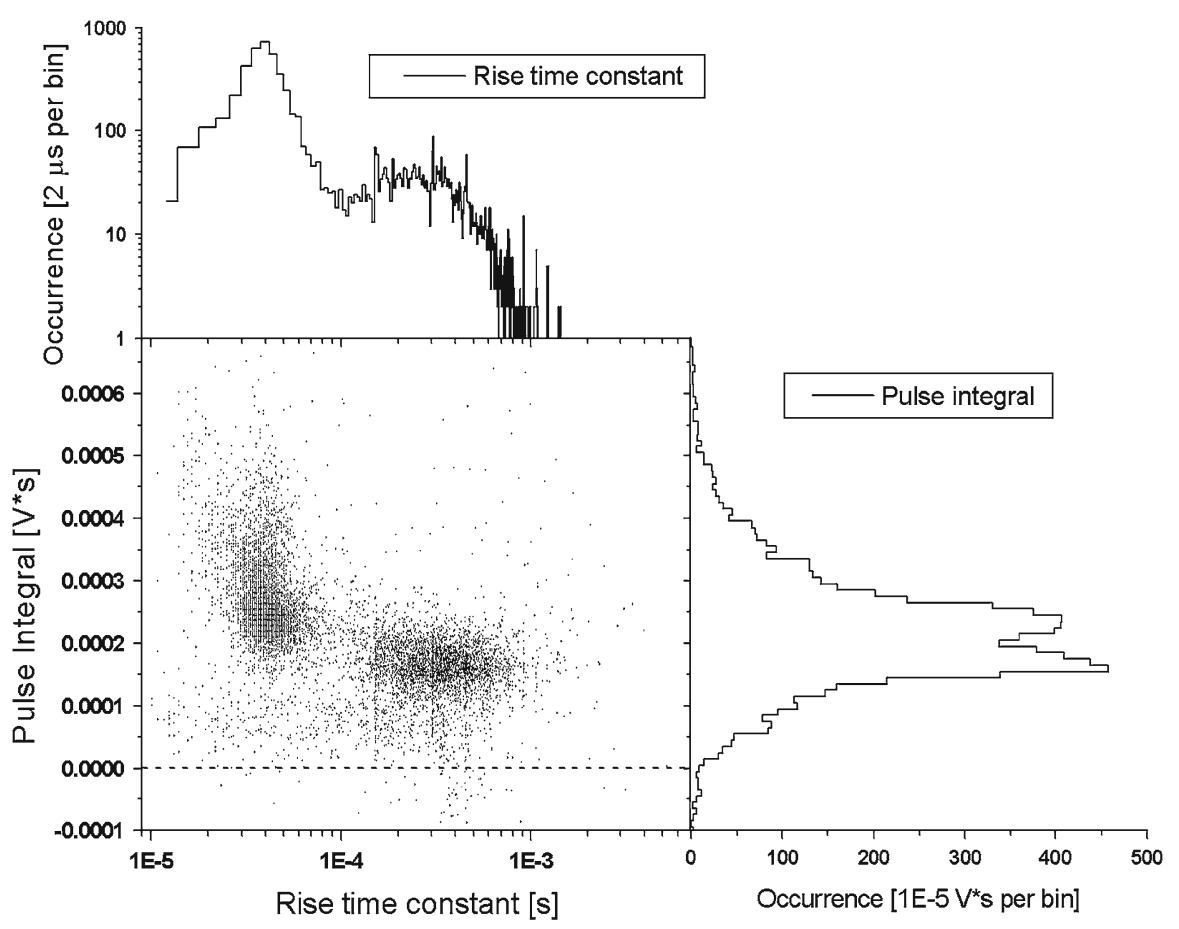

Fig. 4 AC-S3: pulse integral vs rise time constant $\left({ }^{241} \mathrm{Am}\right.$ source $\left.-60 \mathrm{keV}\right)$

is the reference model. To conclude this section, it is worthy of note that also the NASA/GSFC team [15] is developing a cryogenic ACD (121 TES in parallel wired) based on superconducting fins, the main difference with respect to us being related to the TES (Mo/Au instead of Ir). A lot of work has been done by their team to characterize the quasiparticles recombination inside the fins.

\subsection{What We have to Do}

The last part of the previous sub-section calls for further developments. To increase the Athermal phonons collecting efficiency, and to achieve large area pixels for the ATHENA+ design we are following two parallel approaches (see Fig. 5): (a) left panel improve upon Al purity, which translates in a measurement of quasi-particle diffusivity inside the already produced Al strip ( $4 \mathrm{~mm}$ long), shielded by a copper foil with holes corresponding to different positions on the strip, to correlate the rise time and pulse height behaviour with distance from the TES; (b) right panel design a pattern of TES/Al short collecting fingers to maximize the collection of quasi-particles: AC-S6 produced with $1 \mathrm{~cm}^{2}$ area now under testing.

There are other items to be investigated towards the final configuration of the ATHENA+ CryoAC. To properly operate the detector, we have to take into account that the X-IFU thermal reference is set to $50 \mathrm{mK}$. Our idea to use an intermediate stage 

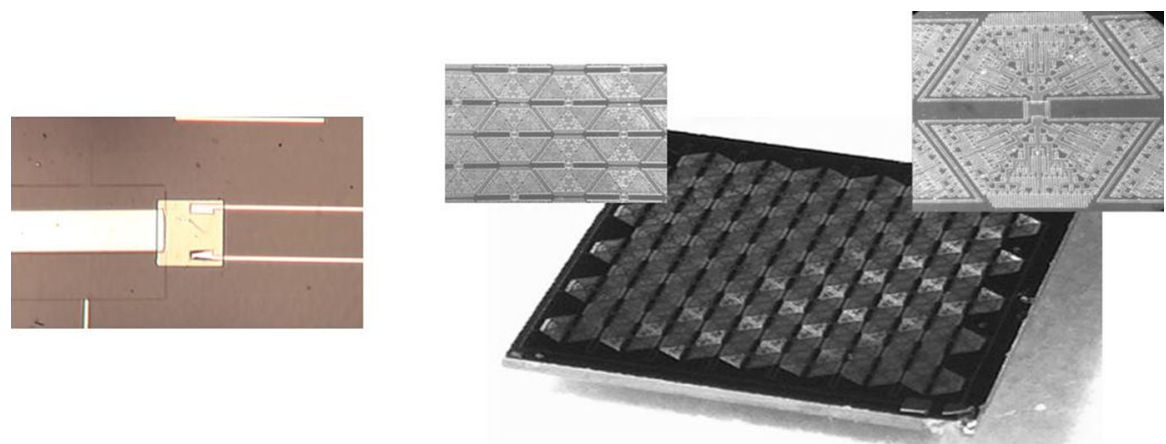

Fig. 5 Left: the sample for the QPs test. The QPs along the Al strip are detected by the TES. Right: the AC-S6 sample (Color figure online)

(called "thermal buffer") between the CryoAC pixel-absorber and the thermal bath, gives us the possibility to avoid the tuning of the transition temperature with respect to the X-IFU one and, more important, to damp the expected very fast athermal rise-front that can induce instabilities in the readout system (SQuID unlocking/flux jump due to the maximum slew rate exceeded and signal amplitude larger than the quasi-linear flux-range of the SQuID [16]). Indeed, to guarantee a thermal decay time faster than the $\mathrm{X}$-IFU one $(\sim 300 \mu \mathrm{s})$ for $\mathrm{T}_{b}=50 \mathrm{mK}, \mathrm{T}_{0} \sim 100 \mathrm{mK}$, typical $\alpha=50$ and $\mathrm{C} \sim 100-150$ $\mathrm{pJ} / \mathrm{K}$, this translates in less than $1 \mathrm{nW}$ of TES bias power. If the TES is typically biased at $10-20 \% \cdot R_{N} \sim 0.02 \mathrm{Ohm}$, we get a TES polarization current of $\mathrm{I}_{b} \sim 200 \mu \mathrm{A}$. Roughly, the expected maximum current-pulse height (i.e. $\Delta E=0.5 \mathrm{MeV}$ at the top of the energy bandwidth) is $\Delta I_{t h} \sim\left(\alpha I_{b} / C T_{0}\right) \cdot \Delta E \sim 60 \mu \mathrm{A}$. The current pulse height of the athermals can be higher than the thermal one just evaluated (see Fig. 3 top-left, and Ref. [10]). So, considering the thermal amplitude as less "alarming case", if we take into account $1 \mu \mathrm{s}$ for the athermal rise time (measured from AC-S1 [17]), we get a pulse slew rate of about $60 \mathrm{~A} / \mathrm{s}$ (i.e. $60 \mu \mathrm{A}$ at the maximum energy over $1 \mu \mathrm{s}$ ) which is quite high since this evaluation accounts for the thermal component. This dynamics, coupled to the expected current pulse height (several tens of $\mu \mathrm{A}$ ) which exceeds the typical maximum value of the quasi-linear flux-range of SQuIDs (in terms of current about some $\mu \mathrm{A}$ ), induces instabilities. Since in the CryoAC environment it is expected a particle count rate [3] of about $5 \mathrm{cts} \mathrm{cm}^{-2} \mathrm{~s}^{-1}$, we derive a rate of unlocking of several time per second. This pulse can be effectively damped by a superconductive coil inserted in series to the TES, or by decreasing the $\mathrm{I}_{b}$ by means of a heater $\left(\mathrm{P}_{H}\right)$ : $I_{b}=\sqrt{\left(G \Delta T-P_{H}\right) / R_{0}}$. At present, we choose the second option because it gives more flexibility to the system, not only for the slew rate but also to vary at convenience the bath temperature. So, the thermal buffer becomes the "new" thermal bath, and by proper thermal conductance values [14] between the absorber and the buffer (GAbsBuffer) and between the buffer and the thermal bath (GBufferBath) we can increase the buffer temperature to the desired value by a heater deposited on it. The samples AC-S2, AC-S3,4, AC-S5 are already be developed placing a silicon-thermal buffer at the absorber bottom connected by $\mathrm{MgO}$ posts. The next two actions to be followed are: (1) to implement the heater; (2) to upgrade the realization of the $\mathrm{MgO}$ posts, now 


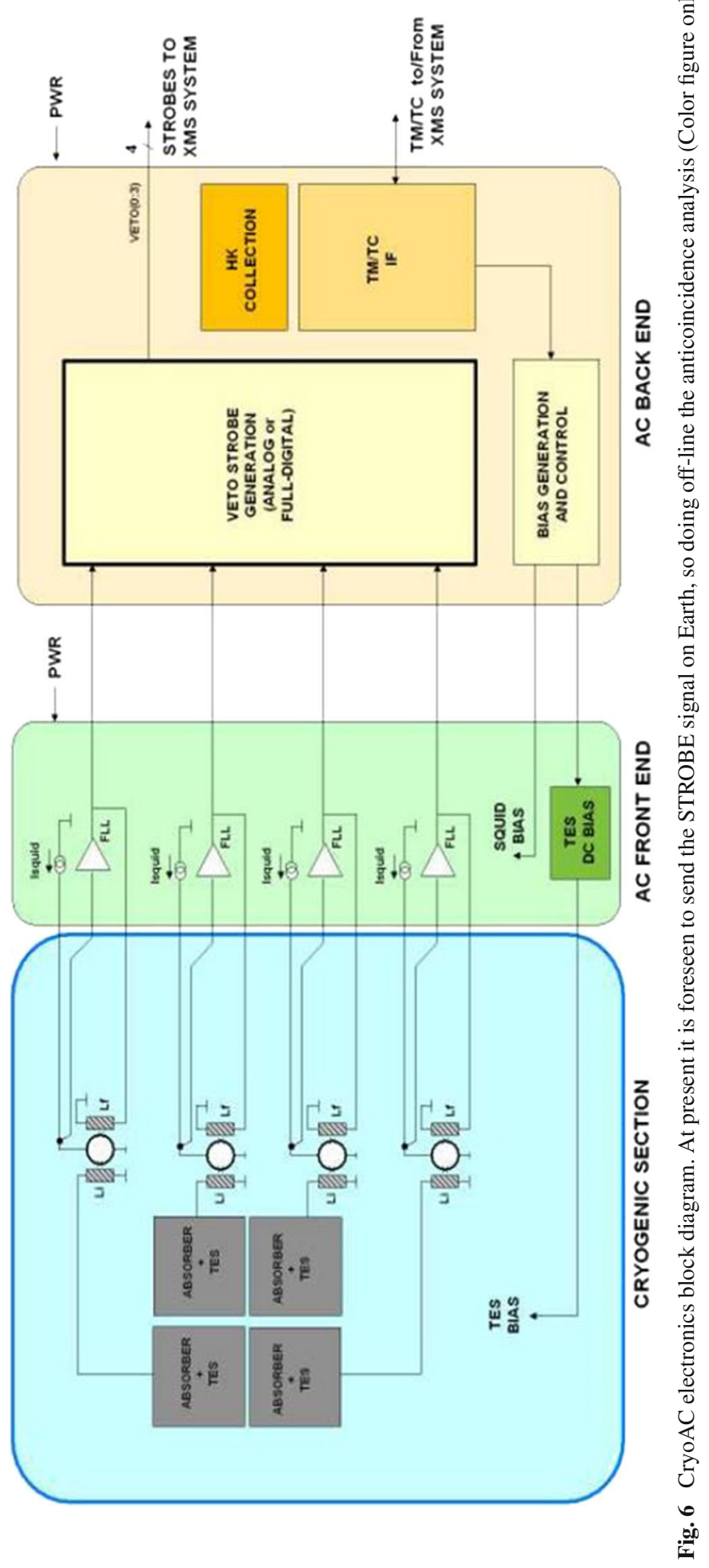


Table 2 Resources for the FLL and back end circuitries

\begin{tabular}{lll}
\hline Budget parameter & Value & Remarks \\
\hline Area/FLL & $<9,000 \mathrm{~mm}^{2}$ & $\begin{array}{c}\text { May increase if heavy RF } \\
\text { filters needed on board }\end{array}$ \\
Power/FLL & $<500 \mathrm{~mW}$ & \\
Mass/FLL & $<100 \mathrm{~g}$ & Excluding connectors \\
Area/BE & $<25,000 \mathrm{~mm}^{2}$ & \\
Power/BE & $<1,700 \mathrm{~mW}$ & TBC \\
Mass/BE & $<300 \mathrm{~g}$ & Excluding connectors \\
\hline
\end{tabular}

directly grown on the Si surface with a cylindrical geometry, so to have a calibrated GAbsBuffer. In conclusion, we remark that deep analysis is ongoing to understand to what extent it is possible to optimize the detector and the related SQuID readout electronics in order to avoid instabilities, along with a possible re-definition of some requirements (e.g. softening the decay time constant specification).

\subsection{The CryoAC Electronics: A Brief Overview}

The schematic diagram concerning the electronics implementation is shown in Fig. 6. The present baseline assumes that the CryoAC detector assembly is built as four identical detectors which cover the area of interest just below the X-IFU. Each of the four detectors is DC-voltage biased and readout by a dedicated SQUID. There is no multiplexing of the four pixels: they are included in a standard analog FLL circuit with $<1.0 \mathrm{MHz}$ bandwidth. The front end electronics, which includes the FLL circuits and the SQUIDs and TESs biasing circuitry, is all in the warm (i.e. no cryo-LNA to be developed). This electronics should be placed as close as possible to the cryostat, possibly inside the same front end box which is foreseen for the X-IFU. An alternative solution could be the development of a dedicated CryoAC front end box.

The back end electronics includes the pulse detection system, the VETO signals generator (VETO LOGIC) and a simple control logic. Also this electronics could be implemented either as a single board hosted in the digital electronics (DE) X-IFU box or as a dedicated small-size unit. About the area/mass/power allocation, the FLL circuitry is deemed not critical in terms of noise and bandwidth considering the recently available space-qualified analog components. Also the back end circuitry should be not critical as it is foreseen to implement it around a FPGA to include all the main digital functions. Table 2 shows a preliminary budget allocation.

\section{Conclusions}

The CryoAC detector for the ATHENA+ mission is a robust program well divided in designing/development/testing activities on samples of TES microcalorimeters and electronics. We have developed several samples to better understand the physics involved in the impulsive response generation. Test results coupled to modelling are 
giving us the information-frame for the design and development of the optimized CryoAC detector for the ATHENA+ X-IFU.

Acknowledgments The authors acknowledge support from the INAF/IAPS and ASI contract (I/035/10/0). $\mathrm{CM}$ also acknowledges Jörn Beyer for useful discussion about the SQuID dynamics. The anonymous referees are acknowledged for having improved this proceeding.

\section{References}

1. http://athena2013.irap.omp.eu/WP/The_Hot_and_Energetic_Universe.pdf. Accessed 10 Mar 2014

2. http://athena2.irap.omp.eu/IMG/pdf/SP_XIFU_APH.pdf. Accessed 10 Mar 2014

3. S. Lotti et al., NIM A 686, 31 (2012)

4. S. Lotti et al., Proc. of SPIE 8443, 84435H-1 (2012)

5. https://twiki.cern.ch/twiki/bin/view/Geant4/LowEnergyElectromagneticPhysicsWorkingGroup. Accessed 10 Mar 2014

6. E. Perinati et al., J. Low Temp. Phys. 167, 232 (2012)

7. R. Gobat et al., A\&A 526, A133, (2011)

8. S. Lotti et al., A\&A to be submitted, (2013)

9. L. Colasanti et al., AIP Conf. Proc. 1185, 438 (2009)

10. F. Pröbst et al., J. Low Temp. Phys. 100, 69, (1995)

11. M. Loidl et al., NIM A 465, 440 (2001)

12. D.S. Akerib et al., Phys. Rev. D 72, 052009 (2005)

13. C. Macculi et al., J. Low Temp. Phys. 167, 783 (2012)

14. C. Macculi et al., Proc. of SPIE 8443, 84435G-1 (2012)

15. C.N. Bailey et al., J. Low Temp. Phys. 167, 236 (2012)

16. Beyer J., Private Communication (PTB-Berlin). (0000)

17. C. Macculi et al., Proc. of SPIE 7732, 77323Y-1 (2010) 